\title{
Research on the application of 3D printing and BIM technology in the direction of bridges
}

\author{
Jiang Zonglin ${ }^{1, *}$ \\ ${ }^{1}$ School of Civil Engineering, Central South University, Changsha, Hunan, China
}

\begin{abstract}
Due to the multi-purpose and multi-collaboration nature of BIM technology, it makes the application of BIM not only the presentation of the structural model, but also the related construction progress and the embodiment of the construction process. It is this multi-functional use that makes BIM technology popular in the industry. 3D printing technology, on the other hand, when used in the field of engineering and construction, mainly prints out parts or all of the structure, and also ensures accuracy and speed. Linking the two will make the future of the related industry in a sudden development. Based on this, this paper will conduct an in-depth study on the application of 3D printing and BIM technology in the direction of bridges, aiming to promote the integration of the civil engineering field with the intelligent manufacturing scene and lay a solid foundation for the construction of modern society.
\end{abstract}

\section{Introduction}

With the development and application of modern transportation has received unprecedented attention, which has greatly facilitated national travel and economic development, and this is the manifestation of China's scientific and technological strength. As we all know, BIM contains a digital model of geometric and nongeometric information of the whole life cycle or some stages of the building. Its ability to gain technical recognition globally and its rise in many countries has drawn the attention of several industries, and our country has started to apply it in the construction, transportation, municipal and electric power industries. At the same time, the emerging 3D printing technology in our construction and transportation industries is a groundbreaking development, a technology that enables the production of set models to be printed under certain procedures. 3D printing technology greatly reduces the manual workload and can even reduce the use of some materials in mature cases. This paper will make an introduction to the construction of bridges in modern transportation and point out the use of BIM+3D technology to provide a reference for the study of modern bridge construction in China. The development of modern technology, bridges are becoming more and more novel in form and complex in structure, which brings a considerable challenge to our traditional industry, and the emerging BIM technology is a product of such an opportunity [1].

\section{BIM technology research related to bridges}

\subsection{Application of BIM technology in the design of assembled bridges}

In the design of assembled bridges, relevant designers should rationalize the pre-burial and pre-preparation process of prefabricated components, and close cooperation and communication among designers are required when carrying out this work. BIM technology is used to build a standardized and comprehensive design platform, and through the communication and collaboration of designers, the information knowledge of the assembled building is effectively integrated, and the problems in the scheme are timely modified and adjusted.

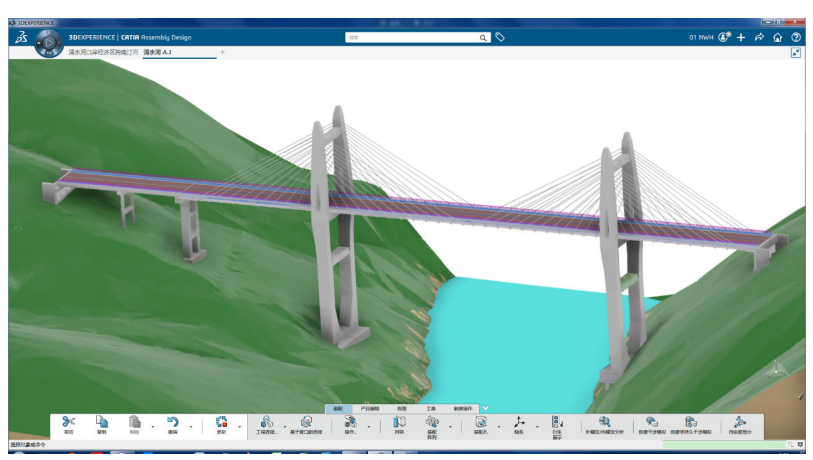

Fig. 1. BIM bridge design drawing (Image from Dasso system)

The design managers integrate BIM technology and "cloud technology" effectively, and transfer the BIM

\footnotetext{
* Corresponding author: zonglin@csu.edu.cn
} 
model information to the BIM integrated design platform within an effective time, with the internal collision and automatic error correction function in BIM technology, it can reduce the problems in professional design. There are many kinds of prefabricated components inside the assembled bridges, and the number of drawings is also relatively large. Using the "collaborative" design in BIM technology can ensure the synchronization of information of designers, and also facilitate the professional designers to make timely improvement and adjustment for the design scheme.

\subsection{The practical use of BIM technology in the production of prefabricated components}

Upgrading the relevant production level: The production process of prefabricated components in assembled bridges occupies an important position in the assembly production. In order to better ensure the accuracy of information in the production of precast components, manufacturers of precast components need to study the BIM models of assembled bridges, extract the dimensional data information, integrate various factors, and construct the parts production planning scheme. In order to better improve the quality of prefabricated components, manufacturers need to incorporate more detailed geometric information, material categories and installation locations in different stages and categories of prefabricated component production, and to rationalize the management of prefabricated components using the technology related to information data RFID chips, so as to better improve the quality of storage and transportation of prefabricated components [2].

Promote research visualization: In order to better improve the quality of assembly bridge projects, designers need to communicate a variety of precast component data information in the BIM model and precast component-related manufacturer information, to better facilitate more manufacturers to understand the product details, dimensions and precast component internal steel grade information parameter data, and information data involved can be converted into barcode way Information parameters, effective integration of precast data information in the BIM model and the assembly bridge precast system, so that the assembly bridge precast production to achieve its intelligent development. Also, the BIM model of the assembled bridge can be printed out by using 3D printing to effectively promote the development of assembled bridge manufacturing [3].

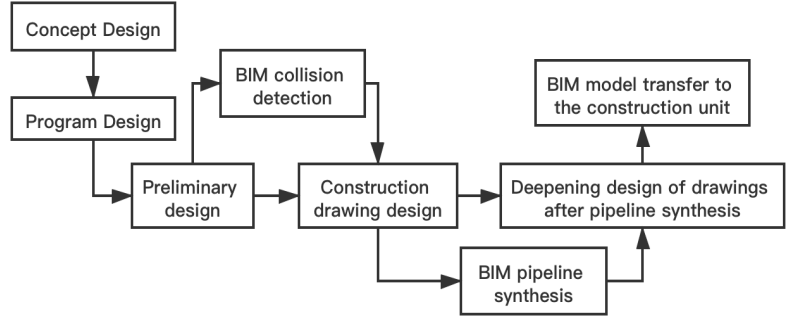

Fig. 2. BIM design flow chart

\section{3D printing technology research related to bridges}

\subsection{Examples of 3D printing technology in bridges}

The famous San Francisco-Oakland New Bay Bridge in the U.S. was built after the partial collapse of the eastern truss bridge during the 1989 San Francisco Bay Area Earthquake, and the California government decided to build a new Bay Bridge to replace the existing eastern truss bridge. The bridge was rebuilt to a seismic intensity of 8 degrees and is considered to be the most expensive bridge in the world, with the highest seismic technology and the longest life expectancy (150 years) to date. It is worth mentioning that, during the reconstruction, the bridge tower was added to the design of shear energyconsuming connection. In the first stage of the test, the bolts broke and the shear deformation zone was not affected by the failure of the bolted connection because it was outside the bolted connection zone. Then cracks appeared first in the longitudinal fillet weld connecting the middle stiffening rib and the flange. The cracks continue to develop in the web as the lateral displacement increases. The brittle damage in the shear joint area appeared. Cyclic loading tests showed that both joint specimens were able to achieve plastic rotation angles in excess of twice that specified for the safety assessment seismic event. However, the plastic rotation angle that developed in the test from the highly confined region where several welds intersected and the brittle cracking of the web resulted in failure to meet the provisions of the AISC I 2002 Seismic Design Code.

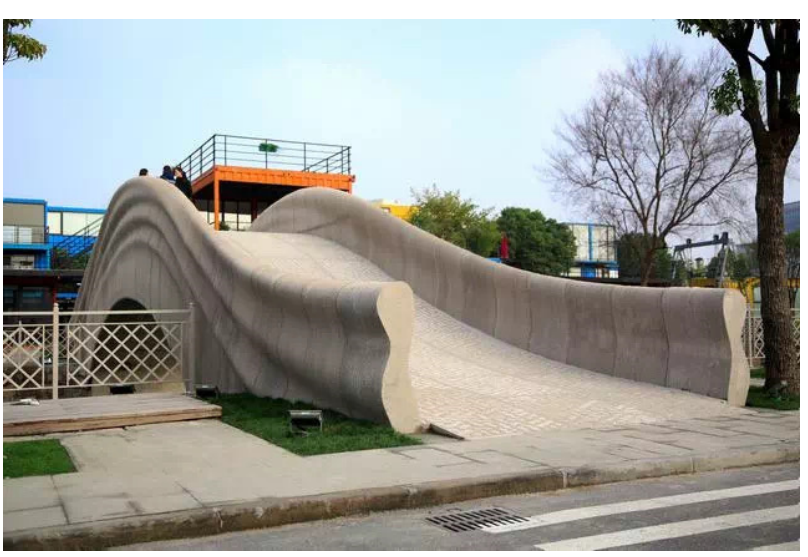

Fig. 3. Chinese Netflix 3D printed bridge (Image from prefabricated building network)

It can be seen that for this type of shear energy dissipation member, the crack also develops from the weakest weld, which causes brittle cracking of the web leading to insufficient plastic rotation capacity, resulting in reduced energy dissipation in earthquakes. This defect can be remedied by using 3D monolithic printing method and the printed shear joint can have greater ductility and plastic deformation capacity due to the uniform material and no large initial defects and stress concentration areas, which is more valuable in seismic applications. In addition to good integrity and improved ductility, 3D 
printing can also replace the external stiffening ribs with internal stiffening ribs for shear energy dissipation connections that are more aesthetically pleasing and space efficient, an advantage that is difficult to reach with traditional welding processes. This advantage is difficult to reach for some traditional welding processes, such as inside the box section members. This advantage is useful for some areas that are difficult to access by traditional welding processes, such as inside box sections. By 3D printing, other high stress areas of shear energy dissipation connections, such as the flange and end plate connections, can be made. The reinforcing flange with curved varying thickness is produced, thus reducing the stress and avoiding premature fracture damage.

\subsection{Exploring the problems in 3D printing applications}

- Evaluation of the adaptability of new materials to 3D printing: With the continuous invention of new materials, various new materials are gradually being introduced into the 3D printing industry. First, the applicability of these materials for 3D printing should be studied and evaluated, that is, the properties of the materials, particle size, powder particle fusibility, thermal and optical characteristics. This series of material properties will become the bottleneck to achieve the mechanical properties, feature resolution, processing accuracy and surface quality required by $3 \mathrm{D}$ printed products.

- Research on the material properties and mechanical properties of 3D printed components: 3D printing produces components that are stacked by layers, and their material composition and properties are different from those of traditionally processed components, so corresponding material properties tests are also needed to obtain various material indicators and mechanical properties of 3D printed components.

- Research on the scale of 3D printing: Since the majority of civil engineering products are large scale (compared to 3D printing has mature applications of medical devices, aviation canine field), 3D printing can broaden the scale of its production to a certain extent determines the breadth of its application, and in the field of large scale can maintain its advantages, this issue is worth exploring.

- The study of the connection between 3D printed parts and the main structure: including the strength, ductility and the way of connection.

- Research on 3D printing of mixed materials or added materials: for example, a difficult problem in steel structures is the resistance to rust, if we can use 3D metal printing in the easy to rust parts and add rust-resistant elements will extend the life of the steel structure, but need to further study the impact of printing of mixed materials on the performance of the components. The application of 3D printing in complex steel structures. Possible applications of 3D printing in complex steel nodes, cast molds, seismic energy-consuming components, building construction formwork, and intelligent construction are presented. Finally, the pre-study of 3D printing in civil engineering applications is discussed to make the realization of its application more realistic.

\section{BIM+3D printing technology related research}

\subsection{D printed concrete technology}

The technology developed on the basis of 3D printing technology applied to concrete construction is called 3D printed concrete technology. It is a new application technology that combines 3D printing technology with the technology in the field of commercial concrete. The main principle is to use $3 \mathrm{D}$ printers to extrude the digital information obtained from 3D modeling through the nozzle of the printer to form concrete components by printing the concrete mix in layers. 3D printing concrete technology can print out complex components, in the actual construction of the printing process can be formed without a mold, in shortening the product manufacturing cycle, improve production efficiency, reduce costs, energy saving and environmental protection has advantages, can make up for the shortcomings of assembled buildings.

\subsection{The integration of BIM and 3D printing technology}

The application of 3D printing technology in the construction industry is undoubtedly a subversion of the traditional initiative, 3D printing assembly building can be achieved by the core of BIM technology. The effective integration of BIM technology and 3D printing technology, each taking advantage of the advantages of information technology, is a unique new theory and method formed in the process of adapting to future development in the construction industry.

The key to the implementation of BIM+3D technology is the need to break the barrier between BIM technology and $3 \mathrm{D}$ printing technology. BIM provides building model information, and 3D printers follow the corresponding path to print. In order to enable BIM data to be input to a $3 \mathrm{D}$ printer, it is necessary to convert the $\mathrm{BIM}$ data into a $3 \mathrm{D}$ printer data code to realize the industrialized production process of prefabricated buildings. Corresponding component information will also be changed in the data processing system. After the path planning is completed, the three-dimensional model composed of all graphic elements can be visually displayed in the BIM software to improve the design plan in time and further modify and monitor the printing process.

The operation of the $3 \mathrm{D}$ printing device is done by the control system, which generates the printing program code to drive the control system after the path planning is completed. The specific process is as follows: the pumping device with the building materials (concrete mix) is driven by the nozzle through the operation of the robot arm, and the instructions of the BIM model are printed layer by layer according to the input printing 
code, forming prefabricated components or directly generating the actual building. Real-time data during the operation of the control system will also be transmitted to the BIM management system to achieve dynamic and visual management of safety, cost, quality and progress during the production of $3 \mathrm{D}$ printed assembled buildings.

\section{Difficulties and suggestions of BIM+3D technology in the application of assembly building}

\subsection{Relevant technical standards are not perfect}

The development process of the integration of BIM technology and 3D printing technology is also in its infancy and still faces many problems. So far, domestic technical standards regarding the integration of BIM and 3D printing technologies are lacking. BIM-related national standards have been issued. All regions, industries, and enterprises are aware of the basic direction and strategy of BIM development. If the entire industry participates in the establishment and implementation of local standards, it can promote the development of construction industry informatization and accelerate $\mathrm{BIM}+3 \mathrm{D}$ printing technology has introduced a higher level of application standards.

\subsection{Lack of information collaboration and interaction platform}

BIM can realize the communication and sharing of building information. It is realized through a collaborative platform during the BIM operation. However, the current interface program between 3D printers and BIM information is lacking, and there is no professional software platform to match it. Due to the different types of data files used between existing BIM platforms and platforms, at the application level, there is only a pointto-point one-way mode, and there is a lack of interactivity between platforms. Therefore, increasing the software and hardware research and development of BIM and $3 \mathrm{D}$ printer platforms is an urgent problem to be solved. It is necessary to continue to introduce a unified standard between the two, only when all participants in the construction industry at all stages use unified standard data for data Interaction can guarantee the uniqueness and continuity of data information in each chain [5].

\subsection{Industry recognition needs to be improved}

Incorporating more novel $3 \mathrm{D}$ printing technology into BIM technology, although it has received strong support from the state, it is not an easy task to be unanimously recognized by investors, designers, constructors, manufacturers and many other industry participants. In addition to insufficient exploration of BIM itself, it is necessary to balance multiple interests, as well as factors such as the risk and uncertainty of future returns. In addition, 3D printing technology currently has a "bottl- eneck" in material development. It lacks experi-mental data such as compression resistance, crack resistance, and impact resistance of related materials. The safety of houses built using 3D printing technology is also an obstacle to public acceptance and recognition.Therefore, it is necessary to continue to increase the research and development of $\mathrm{BIM}+3 \mathrm{D}$ printing technology, such as in-depth research on waste materials such as construction waste to realize the green of printing materials: color construction, and adding fiber materials to concrete substrates to improve its seismic performance. In addition, expand the training of industrialized technology and professional talents familiar with prefab-ricated buildings, actively explore and try new BIM applications in engineering applications, and sum up experience in more practices, so as to accelerate the construction industry' s BIM+ Promotion of 3D technology.

\section{Conclusion}

The impact of new technologies in the field of construction on the future is very important. The present is the information age, and the main characteristics of the information age will inevitably permeate all aspects of future architecture. BIM+3D technology can control quite high precision in the design and construction of bridges, which also meets the requirements of local regulations and has a great positive effect. Coupled with the integrity of BIM technology, the time control of the whole process, the diversity of information, the unity, etc., it brings great convenience to our project. In short, the application of new technologies such as BIM and 3D printing in the field of construction has brought great benefits. Therefore, we not only need to develop our BIM technology and 3D printing technology in the construction industry, but also we should perfectly combine the two technologies, develop together, and maximize benefits.

\section{References}

1. Li H, Chen Z. (2019) Research progress of prefabricated buildings based on $\mathrm{BIM}+3 \mathrm{D}$ printing technology. Construction Technology, 48: 276-279.

2. Zhang W, Liu X. (2019) Exploration of BIM and 3D printing application mode. Value Engineering, 38: 225-227.

3. Zhang Y. (2018) Problems and countermeasures in the application of 3D printing technology in the construction industry. Construction Economics, 39: 10-13.

4. Fan D, Xu S, Cui T. (2018) Application of 3D printing technology in bridge model. Science and Technology Innovation, 1:136-137.

5. Tao Y, Zhang Y, Chen Y, et al. (2014) Prospects of 3D printing technology in civil engineering. Steel Structure, 29: 1-8. 